\title{
EXTENSIONS OF A THEOREM OF JORDAN ON PRIMITIVE PERMUTATION GROUPS
}

\author{
MARTIN W. LIEBECK
}

(Received 18 August 1980; revised 4 December 1981)

Communicated by D. E. Taylor

\begin{abstract}
Let $G$ be a primitive permutation group of finite degree $n$ containing a subgroup $H$ which fixes $k$ points and has $r$ orbits on $\Delta$, the set of points it moves. An old and important theorem of Jordan says that if $r=1$ and $k \geqslant 1$ then $G$ is 2-transitive; moreover if $H$ acts primitively on $\Delta$ then $G$ is $(k+1)$-transitive. Three extensions of this result are proved here: (i) if $r=2$ and $k \geqslant 2$ then $G$ is 2-transitive, (ii) if $r=2, n>9$ and $H$ acts primitively on both of its two nontrivial orbits then $G$ is $k$-primitive, (iii) if $r=3, n>13$ and $H$ acts primitively on each of its three nontrivial orbits, all of which have size at least 3 , then $G$ is $(k-1)$-primitive.
\end{abstract}

1980 Mathematics subject classification (Amer. Math. Soc.): 20 B 20.

\section{Introduction}

In 1871 Jordan proved the following result (see Theorem I of Jordan (1871) or Theorems 13.1, 13.2 of Wielandt (1964)): let $G$ be a primitive permutation group on a finite set $\Omega$ and suppose that $G$ has a subgroup $H$ which fixes at least one point of $\Omega$ and is transitive on $\Delta=\operatorname{supp}(H)$, the set of points not fixed by $H$. Then $G$ is 2-transitive; moreover if $H$ is primitive on $\Delta$ then $G$ is $(k+1)$-fold transitive where $k=\mid$ fix $H \mid$.

This result has played a large part in the study of permutation groups since then (see for instance $\$ 13$ of Wielandt (1964)). In fact Theorem I of Jordan (1871) gives more information than stated above: $G$ is $(k+1)$-fold transitive provided that $H$ is transitive on $\Delta$ and for all $f \geqslant 1, H$ admits at most one congruence on $\Delta$ of modulus $f$. Marggraff (1892) generalised this, showing that the same conclusion

C. Copyright Australian Mathematical Society 1983 
holds if $H$ is transitive on $\Delta$ and for all $f \geqslant 1, H$ admits at most $f$ congruences on $\Delta$ of modulus $f$.

Two questions which arise from these results are: (a) what can be said about a primitive group $G$ which has a subgroup $H$ having $r$ orbits on $\operatorname{supp}(H)$, where $r>1$ ? (b) what more can be said if we make assumptions about the action of $H$ on its orbits? The purpose of this paper is to give some answers to these questions in the cases $r=2$ and $r=3$. For $r=2$ we prove Theorems 1 and 2:

THEOREM 1. Let $G$ be a primitive group of degree $n$ on a finite set $\Omega$ and suppose that $G$ has a subgroup $H$ fixing at least two points of $\Omega$ and having two orbits $\Sigma_{1}, \Sigma_{2}$ on $\operatorname{supp}(H)$. Then $G$ is 2-transitive.

THEOREM 2. If, with the hypotheses of Theorem $1, H$ is primitive on $\Sigma_{1}$ and on $\Sigma_{2}$ and $\left|\Sigma_{1}\right| \geqslant 3,\left|\Sigma_{2}\right| \geqslant 3$ then one of the following holds:

(i) $G$ is $k$-fold primitive (where $k=\mid$ fix $H \mid$ ),

(ii) $n=9, k=3$ and $G$ is $A S L(2,3)$ or $A G L(2,3)$.

Theorem 2 is best possible, for there are many simply primitive groups $G$ in which the stabiliser $G_{\alpha}$ has two nontrivial orbits and acts primitively on both. The hypotheses of Theorem 2 are also considered in Antopolski (1971), where such a group $G$ is shown to be 2-transitive.

For the case $r=3$ we prove:

THEOREM 3. Let $G$ be primitive on a finite set $\Omega$ with a subgroup $H$ such that $\mid$ fix $H \mid=k$ and $H$ has 3 orbits $\Sigma_{1}, \Sigma_{2}, \Sigma_{3}$ on $\operatorname{supp}(H)$. Suppose that $H$ acts primitively on each $\Sigma_{i}$ and $\left|\Sigma_{i}\right| \geqslant 4(i=1,2,3)$. Then $G$ is $(k-1)$-fold primitive.

Again Theorem 3 is best possible, for there is a simply primitive group $G$ in which $G_{\alpha \beta}$ has three nontrivial orbits and acts primitively on each of them (see Example 3.3). The general cases $r=3, r=4, r=5$ are considered in Liebeck (1977), where it is proved that $G$ has rank at most $r+1$, thus verifying a conjecture of Wielandt (1971) in these cases. It is very likely that the methods of this paper will extend to prove further results for small values of $r$.

This paper is divided into four sections. In the first Theorem 1 is proved; the proof relies heavily on the graph theory associated with a permutation group as described in Neumann (1977). The second section consists of a proof of Theorem 2 ; here a different approach is taken - the orbits $\Sigma_{1}, \Sigma_{2}$ of $H$ are 'built up' step by step until they become orbits of $G_{\alpha}$ for some $\alpha \in \Omega$. Then a theorem of O'Nan (1975) on 2-transitive but not 2-primitive groups is used to complete the proof. The proof of Theorem 3 , given in the fourth section, runs along similar lines. In Section 3 some examples of primitive groups having subgroups with few orbits and several fixed points are presented. 
The notation used is that of Wielandt (1964), except that for a subset $\Delta$ of $\Omega$ we write $G_{(\Delta)}, G_{\{\Delta\}}$ for the pointwise and setwise stabilisers of $\Delta$ in $G$, respectively.

\section{Proof of Theorem 1}

Before proving Theorem 1 we briefly outline the results in Neumann (1977) which we shall need. Let $G$ be a transitive permutation group on a finite set $\Omega$ and let $\alpha \in \Omega$. There is a 1-1 correspondence between the orbits $\Delta_{0}, \Delta_{1}, \ldots, \Delta_{s}$ of $G$ on $\Omega \times \Omega$ and the orbits $\Gamma_{0}, \Gamma_{1}, \ldots, \Gamma_{s}$ of $G_{\alpha}$ on $\Omega$ given by

$$
\Gamma_{i}=\Gamma_{i}(\alpha)=\left\{\gamma \in \Omega \mid(\alpha, \gamma) \in \Delta_{i}\right\} .
$$

For $i \geqslant 1$ we define the $\Gamma_{i}$-graph to be the directed graph on $\Omega$ in which there is an edge from $\beta$ to $\gamma$ if and only if $(\beta, \gamma) \in \Delta_{i}$. We denote by $\Gamma_{i}^{*}$ the suborbit paired with $\Gamma_{i}$. If $\Gamma_{i}$ is self-paired then the $\Gamma_{i}$-graph is undirected.

Suppose now that $G$ is primitive on $\Omega$. Then all the $\Gamma_{i}$-graphs are connected (Lemma 3 of Neumann (1977)). For any $j, k$ define

$\Delta_{j} \circ \Delta_{k}=\left\{(\beta, \gamma) \mid \beta \neq \gamma\right.$ and there exists $\delta \in \Omega$ with $\left.(\beta, \delta) \in \Delta_{j},(\delta, \gamma) \in \Delta_{k}\right\}$.

Let $\Gamma_{j} \circ \Gamma_{k}$ be the corresponding union of suborbits. The following result is taken from Theorems 3 and 4 of Neumann (1977).

Result A. (i) Suppose that for $\beta \in \Gamma_{i}, G_{\alpha \beta}$ is transitive on $\Gamma_{j}$ and that $\left|\Gamma_{i}\right|>1$, $\left|\Gamma_{j}\right|>1$. Then $\Gamma_{i}^{*} \circ \Gamma_{j}$ is a single suborbit of size greater than $\left|\Gamma_{i}\right|$ and $\left|\Gamma_{j}\right|$.

(ii) If $s \geqslant 2$ and $G_{\alpha}$ acts 2-transitively on $\Gamma_{i}$ for some $i \geqslant 1$ then $\Gamma_{i}^{*} \circ \Gamma_{i}$ is a single suborbit of size greater than $\left|\Gamma_{i}\right|$.

ProOf OF THEOREM 1. Let $G$ be a primitive permutation group of degree $n$ on $\Omega$ and assume that $G$ has a subgroup $H$ fixing at least 2 points and having 2 orbits $\Sigma_{1}, \Sigma_{2}$ on $\operatorname{supp}(H)$, the support of $H$ (that is, $\Omega \backslash$ fix $H$ ). Suppose for a contradiction that $G$ is not 2-transitive. Let $X$ be a subgroup of $G$ with $|\operatorname{supp}(X)|$ maximal subject to the following conditions:

(a) $\mid$ fix $X \mid \geqslant 2$,

(b) $X$ has at most 2 orbits on $\operatorname{supp}(X)$.

By Jordan's Theorem, $X$ has precisely 2 orbits $\Delta_{1}, \Delta_{2}$ on $\operatorname{supp}(X)$. Write $\Delta=\Delta_{1} \cup \Delta_{2}$ and $d_{i}=\left|\Delta_{i}\right|(i=1,2)$.

Lemma 1.0. Suppose $g \in G$ is such that $\Delta_{1} g \cap \Delta_{1} \neq \varnothing$ and $\Delta_{1} g \neq \Delta_{1}$. Then $\Delta_{2} g \cap \Delta \neq \varnothing$ and $|\Delta g \cup \Delta| \geqslant n-1$.

Proof. Let $Y=\left\langle X, X^{g}\right\rangle$. The nontrivial orbits of $Y$ consist of unions among the sets $\Delta_{1}, \Delta_{2}, \Delta_{1} g$ and $\Delta_{2} g$. Suppose that $\Delta_{2} g \cap \Delta=\varnothing$. Then the nontrivial 
orbits of $Y$ are either

(i) $\Delta_{2} g$ and $\Delta_{1} g \cup \Delta_{1} \cup \Delta_{2}$ (if $\Delta_{1} g \cap \Delta_{2} \neq \varnothing$ ), or

(ii) $\Delta_{2} g, \Delta_{2}$ and $\Delta_{1} g \cup \Delta_{1}$ (if $\Delta_{1} g \cap \Delta_{2}=\varnothing$ ).

In case (i) we may choose $y \in X^{g}$ such that $\Delta_{1} y \cap \Delta_{1} \neq \varnothing$ and $\Delta_{1} y \cap \Delta_{2} \neq \varnothing$. Let $Z=\left\langle X, X^{y}\right\rangle$. If $|\operatorname{supp}(Z)|=|\operatorname{supp}(X)|$ then $Z$ has just 1 nontrivial orbit, so $G$ is 2 -transitive by Jordan's Theorem, which is not so. Hence $|\operatorname{supp}(Z)|>$ $|\operatorname{supp}(X)|$. However $Z$ has at most 2 nontrivial orbits and certainly $\Delta_{2} g \subseteq$ fix $Z$, so $\mid$ fix $Z \mid \geqslant 2$ and $Z$ contradicts our choice of $X$. In case (ii) we may pick $y \in X^{g}$ with $\Delta_{1} y \cap \Delta_{1} \neq \varnothing$ and $\Delta_{1} y \neq \Delta_{1}$; then $\left\langle X, X^{y}\right\rangle$ gives a similar contradicition.

Hence $\Delta_{2} g \cap \Delta \neq \varnothing$. Finally, suppose that $|\Delta g \cup \Delta| \leqslant n-2$. Then $\mid$ fix $Y \mid \geqslant$ 2. Clearly $Y$ has at most 2 nontrivial orbits, so by choice of $X$ we must have $\operatorname{supp}(Y)=\operatorname{supp}(X)$. But then since $\Delta_{1} g \cap \Delta_{1} \neq \varnothing$ and $\Delta_{1} g \neq \Delta_{1}, Y$ has just 1 nontrivial orbit, so $G$ is 2-transitive by Jordan's Theorem. This contradiction shows that $|\Delta g \cup \Delta| \geqslant n-1$, proving the lemma.

We continue the proof of Theorem 1 in a series of steps.

STEP 1.1. We have $\mid$ fix $X\left|<\frac{1}{2} n<\right| \Delta \mid$. For suppose that $|\Delta| \leqslant \frac{1}{2} n$. Since $G$ is primitive we may choose $g \in G$ such that $\Delta_{1} g \cap \Delta_{1} \neq \varnothing$ and $\Delta_{1} g \neq \Delta_{1}$. By Lemma 1.0 then, $\Delta_{2} g \cap \Delta \neq \varnothing$ and $|\Delta g \cup \Delta| \geqslant n-1$. However

$$
|\Delta g \cup \Delta|=|\Delta g|+|\Delta|-|\Delta g \cap \Delta| \leqslant \frac{1}{2} n+\frac{1}{2} n-2=n-2,
$$

which is a contradiction.

STEP 1.2. Let $\alpha \in$ fix $X$ and let $\Gamma_{1}, \ldots, \Gamma_{s}(s \geqslant 2)$ be the nontrivial orbits of $G_{\alpha}$. Suppose that $\Delta_{1}$ and $\Delta_{2}$ lie in different orbits, say $\Delta_{1} \subseteq \Gamma_{1}, \Delta_{2} \subseteq \Gamma_{2}$. Then $\Delta_{1}, \Delta_{2}$ are blocks of imprimitivity for $G_{\alpha}^{\Gamma_{1}}, G_{\alpha}^{\Gamma_{2}}$ respectively. Further, either $\Delta_{1}=\Gamma_{1}$ or $\Delta_{2}=\Gamma_{2}$.

Note that the last part of Step 1.2 follows from the first, for if $\Delta_{1} \subset \Gamma_{1}$ and $\Delta_{2} \subset \Gamma_{2}$ then by the first part, $\left|\Delta_{i}\right| \leqslant \frac{1}{2}\left|\Gamma_{i}\right|(i=1,2)$, so $|\Delta| \leqslant \frac{1}{2} n$, contradicting Step 1.1.

Suppose that $\Delta_{1}$ is not a block for $G_{\alpha}^{\Gamma_{1}}$ and pick $g \in G_{\alpha}$ such that $\Delta_{1} g \neq \Delta_{1}$ and $\Delta_{1} g \cap \Delta_{1} \neq \varnothing$. By Lemma 1.0, $\Delta_{2} g \cap \Delta \neq \varnothing$ and $\Delta g \cup \Delta=\Omega \backslash\{\boldsymbol{\alpha}\}$, from which it follows that $s=2, \Delta_{1} g \cup \Delta_{1}=\Gamma_{1}, \Delta_{2} g \cup \Delta_{2}=\Gamma_{2}$ and $\Delta_{2} g \cap \Delta_{2} \neq \varnothing$. Let $\Theta_{i}=\Gamma_{i} \backslash \Delta_{i}(i=1,2)$. Then $\Theta_{1} \neq \varnothing$ since $\Delta_{1}$ is not a block for $G_{\alpha}^{\Gamma_{1}}$, so $1 \leqslant\left|\Theta_{1}\right|<d_{1}$.

We complete Step 1.2 in the following stages:

(a) $\Theta_{1}$ is a block for $G_{\alpha}^{\Gamma_{1}}$. For otherwise there exists $h \in G_{\alpha}$ with $\Theta_{1} h \neq \Theta_{1}$ and $\Theta_{1} h \cap \Theta_{1} \neq \varnothing$, forcing $|\Delta h \cup \Delta|<n-1$, which contradicts Lemma 1.0.

(b) $\Gamma_{1}, \Gamma_{2}$ are self-paired suborbits of $G$. For $G_{\alpha}$ acts 2-transitively on the block system for $G_{\alpha}^{\Gamma_{1}}$ which contains $\Theta_{1}$. Hence 2 divides $|G|$ and (b) follows. 
(c) We have $\Delta_{2} \subset \Gamma_{2}$. Suppose on the contrary that $\Delta_{2}=\Gamma_{2}$. Then if $\beta \in \Theta_{1}$ we have $X \leqslant G_{\alpha \beta}$, so $G_{\alpha \beta}$ is transitive on $\Gamma_{2}$. But then by Result $\mathrm{A}, \Gamma_{2} \circ \Gamma_{1}$ is a suborbit of $G$ of size greater than $\left|\Gamma_{1}\right|$ and $\left|\Gamma_{2}\right|$, which is a contradiction.

Hence $1 \leqslant\left|\Theta_{2}\right|<d_{2}$ and as for (a), $\Theta_{2}$ is a block for $G_{\alpha}^{\Gamma_{2}}$. For $i=1,2$ let $9_{i}$ be the block system for $G_{\alpha}^{\Gamma_{i}}$ containing $\Theta_{i}$ and let $k_{i}=\left|\mathscr{R}_{i}\right|$.

(d) We have $G_{\alpha\left\{\boldsymbol{\theta}_{1}\right\}}=G_{a\left\{\boldsymbol{\theta}_{2}\right\}}$. Also $k_{1}=k_{2}=k$ and there exist $g_{2}, \ldots, g_{k} \in G_{\alpha}$ such that $\mathscr{G}_{i}=\left\{\Theta_{i}, \Theta_{i} g_{2}, \ldots, \Theta_{i} g_{k}\right\}(i=1,2)$. For if there exists $h \in G_{\alpha\left\{\Theta_{2}\right\}} \backslash G_{\alpha\left\{\Theta_{1}\right\}}$ then $|\Delta h \cup \Delta|<n-1$, contradicting Lemma 1.0. The last part follows if we take $\left\{1, g_{2}, \ldots, g_{k}\right\}$ to be a set of coset representatives for $G_{\alpha\left\{\theta_{1}\right\}}$ in $G$.

As described at the beginning of this section, for $\beta \in \Omega$ we write $\Gamma_{1}(\beta), \Gamma_{2}(\beta)$ for the nontrivial orbits of $G_{\beta}$ (so $\Gamma_{1}=\Gamma_{1}(\alpha), \Gamma_{2}=\Gamma_{2}(\alpha)$ ).

(e) One of the following holds:

(i) $\beta \in \Gamma_{1}(\gamma)$ for all $\beta \in \Theta_{1}, \gamma \in \Delta_{2}$;

(ii) $\beta \in \Gamma_{2}(\gamma)$ for all $\beta \in \Theta_{2}, \gamma \in \Delta_{1}$.

For suppose that (ii) is false. Then there exist $\beta \in \Theta_{2}, \gamma \in \Delta_{1}$ such that $\beta \in \Gamma_{1}(\gamma)$. Since $X \leqslant G_{\alpha \beta}$ and $X$ is transitive on $\Delta_{1}$ this means that $\beta \in \Gamma_{1}(\gamma)$ for all $\gamma \in \Delta_{1}$. It follows that $\beta g_{2} \in \Gamma_{1}\left(\gamma g_{2}\right)$ for all $\gamma \in \Delta_{1}$; however $\beta g_{2} \in \Delta_{2}$ and $\Theta_{1} \subseteq \Delta_{1} g_{2}$. Hence $\beta^{\prime} \in \Gamma_{1}\left(\gamma^{\prime}\right)$ for some $\beta^{\prime} \in \Theta_{1}, \gamma^{\prime} \in \Delta_{2}$ and we see that (i) holds by considering the actions of $G_{\alpha\left\{\boldsymbol{\theta}_{1}\right\}}$ and $X$.

We assume without loss of generality that (i) holds.

(f) Let $t_{i}=\left|\Theta_{i}\right|(i=1,2)$. Then $t_{1}>d_{2}$. Suppose that $t_{1} \leqslant d_{2}$. First we show that the $\Gamma_{1}$-graph has no triangles. To do this we choose $\beta \in \Theta_{1}$ and show that $\beta$ is joined in the $\Gamma_{1}$-graph to no point of $\Gamma_{1}$. By (e), $\beta \in \Gamma_{1}(\gamma)$ for all $\gamma \in \Delta_{2}$. If $\beta \in \Gamma_{1}(\delta)$ for some $\delta \in \Delta_{1}$ then $\beta \in \Gamma_{1}(\delta)$ for all $\delta \in \Delta_{1}$ (by the action of $X$ ), so the valency $v_{1}(\beta)$ of $\beta$ in the $\Gamma_{1}$-graph satisfies

$$
v_{1}(\beta) \geqslant d_{1}+d_{2}+1 \text {. }
$$

However $v_{1}(\beta)=\left|\Gamma_{1}\right|=d_{1}+t_{1}$, from which it follows that $t_{1}>d_{2}$, contrary to assumption. Hence $\beta$ is joined in the $\Gamma_{1}$-graph to no point of $\Delta_{1}$. If $\beta \in \Gamma_{1}(\delta)$ for some $\delta \in \Theta_{1}$ then by (e), $\beta$ and $\delta$ have at least $d_{2}$ mutual adjacenices in the $\Gamma_{1}$-graph. However $\alpha \in \Gamma_{1}(\beta)$ and $\alpha, \beta$ have at most $t_{1}-1$ mutual adjacencies in the $\Gamma_{1}$-graph, so $t_{1}-1 \geqslant d_{2}$ which is not so. Hence $\beta$ is joined in the $\Gamma_{1}$-graph to no point of $\Gamma_{1}$ and we have shown that the $\Gamma_{1}$-graph has no triangles.

Now let $\beta^{\prime} \in \Theta_{2}$ and suppose $\beta^{\prime} \in \Gamma_{1}(\gamma)$ for some $\gamma \in \Theta_{2}$. Then $\beta^{\prime} g_{2} \in \Gamma_{1}\left(\gamma g_{2}\right)$ and $\beta^{\prime} g_{2}, \gamma g_{2} \in \Delta_{2}$, so $\beta, \beta^{\prime} g_{2}, \gamma g_{2}$ form a triangle in the $\Gamma_{1}$-graph, which is a contradiction. Since the $\Gamma_{2}$-graph is connected (Lemma 3 of Neumann (1977)) there exists $\delta \in \Gamma_{2}$ with $\beta^{\prime} \in \Gamma_{1}(\delta)$; it must be the case that $\delta \in \Delta_{2}$. Hence $\beta^{\prime} \in \Gamma_{1}(\delta)$ for all $\delta \in \Delta_{2}$.

Finally, if $\beta^{\prime} \in \Gamma_{1}(\gamma)$ for some $\gamma \in \Theta_{1}$ then $\beta^{\prime}, \gamma, \delta$ form a triangle in the $\Gamma_{1}$-graph for any $\delta \in \Delta_{2}$. Hence $\beta^{\prime} \in \Gamma_{1}(\gamma)$ for all $\gamma \in \Delta_{1}$. Consequently

$$
v_{1}\left(\beta^{\prime}\right)=d_{1}+d_{2} \text {. }
$$


But then $v_{1}(\beta)=d_{1}+d_{2}$, so $\beta$ must be joined in the $\Gamma_{1}$-graph to some point of $\Gamma_{1} \cup \Theta_{2}$. This we have seen above to be impossible. This contradiction establishes (f).

Now we can complete Step 1.2. Let $\beta \in \Theta_{1}$. Then $v_{1}(\beta)=\left|\Gamma_{1}\right|=k t_{1}$, and $k \geqslant 3$. By (f) we have $t_{1}>d_{2}>t_{2}$, so

$$
k t_{1} \geqslant t_{1}+2 t_{1}>t_{1}+d_{2}+t_{2}=\left|\Theta_{1}\right|+\left|\Gamma_{2}\right| .
$$

Thus $v_{1}(\beta)>\left|\Gamma_{2} \cup \Theta_{1}\right|$ and so $\beta \in \Gamma_{1}(\gamma)$ for some $\gamma \in \Delta_{1}$, hence for all $\gamma \in \Delta_{1}$. By (e) then, $\beta \in \Gamma_{1}(\gamma)$ for all $\gamma \in \Delta\left(=\Delta_{1} \cup \Delta_{2}\right)$. It follows that for each $j$, $\delta \in \Gamma_{1}(\varepsilon)$ for all $\delta \in \Theta_{1} g_{j}, \varepsilon \in \Delta g_{j}$. Also $\Delta g_{j}=\left(\Gamma_{1} \cup \Gamma_{2}\right) \backslash\left(\Theta_{1} \cup \Theta_{2}\right) g_{j}$. Consequently in the $\Gamma_{2}$-graph, points of $\Theta_{1} g_{j}$ can be joined only to points of $\left(\Theta_{1} \cup \Theta_{2}\right) g_{j}$. Let $\beta \in \Theta_{1}, \gamma \in \Theta_{1} g_{2}$. Then $\beta \in \Gamma_{1}(\gamma)$ and $\beta, \gamma$ have no mutual adjacencies in the $\Gamma_{2}$-graph. However $\gamma \in \Gamma_{1}(\alpha)$ and $\alpha, \gamma$ have at least one mutual adjacency in the $\Gamma_{2}$-graph since the $\Gamma_{2}$-graph is connected.

This final contradiction completes Step 1.2.

StEP 1.3. Let $\alpha \in$ fix $X$ and let $\Gamma_{1}, \ldots, \Gamma_{s}$ be the nontrivial orbits of $G_{\alpha}$. Then each $\Gamma_{i}(i=1, \ldots, s)$ is a union of sets of the form $\Delta_{j} g(j=1$ or 2$)$ where $g \in G$ and $X^{g} \leqslant G_{\alpha}$.

To see this, let

$$
W=\left\langle X^{g} \mid g \in G, X^{g} \leqslant G_{\alpha}\right\rangle .
$$

Then $W \triangleleft G_{\alpha}$ and $W$ is weakly closed in $G_{\alpha}$ with respect to $G$, so by Theorem 3.5 of Wielandt (1964), $G_{\alpha}=N_{G}(W)$ is transitive on fix $W$. Hence fix $W=\{\alpha\}$ and so for any $\gamma \in \Gamma_{i}$ there exists $g_{\gamma} \in G$ such that $X^{g_{\gamma}} \leqslant G_{\alpha}$ and $\gamma \in \operatorname{supp}\left(X^{g_{\gamma}}\right)=$ $\Delta g_{\gamma}$. Step 1.3 follows.

We can now finish the proof of Theorem 1. First suppose that $\Delta_{1}, \Delta_{2}$ lie in different suborbits of $G$, say $\Delta_{1} \subseteq \Gamma_{1}, \Delta_{2} \subseteq \Gamma_{2}$. By Step 1.2 we may assume that $\Delta_{1}=\Gamma_{1}$. Choose $t$ such that $\Gamma_{t} \cap$ fix $X \neq \varnothing$ and

$$
\left|\Gamma_{t}\right|=\max \left\{\left|\Gamma_{i}\right|: \Gamma_{i} \cap \text { fix } X \neq \varnothing\right\},
$$

so that $t \geqslant 2$. Let $\beta \in \Gamma_{t} \cap$ fix $X$; then $X \leqslant G_{\alpha \beta}$. Hence if $\Delta_{2}=\Gamma_{2}$ then by Result A, one of $\Gamma_{t}^{*} \circ \Delta_{1}$ and $\Gamma_{t}^{*} \circ \Delta_{2}$ is a suborbit of $G$ of size greater than $\left|\Gamma_{t}\right|,\left|\Delta_{1}\right|$ and $\left|\Delta_{2}\right|$, which is clearly impossible. And if $\Delta_{2} \subset \Gamma_{2}$ then $\Gamma_{t}^{*} \circ \Delta_{1}$ gives a similar contradiction (for then $\left|\Gamma_{t}\right| \geqslant\left|\Gamma_{2}\right|$ by choice of $\Gamma_{t}$ ).

Thus we may suppose that $\Delta_{1}, \Delta_{2}$ lie in the same suborbit of $G$, say $\Delta_{1} \cup \Delta_{2} \subseteq$ $\Gamma_{1}$. By Step 1.3 there exists $g \in G$ such that $X^{g} \leqslant G_{\alpha}$ and, say, $\Delta_{1} g \subseteq \Gamma_{2}$. Certainly $\left|\Gamma_{2}\right|<|\Delta|$ by Step 1.1, so $\Delta_{2} g \subseteq \Gamma_{t}$ for some $t \neq 2$. Hence $\Delta_{1} g, \Delta_{2} g$ lie in different suborbits of $G$ and now the argument of the previous paragraph yields a contradiction with $X^{g}$ replacing $X$ and $\Delta_{i} g$ replacing $\Delta_{i}(i=1,2)$.

This completes the proof of Theorem 1 . 


\section{Proof of Theorem 2}

Before embarking on the proof of Theorem 2 we prove a lemma necessary to the proof.

LемMA 2.0. Let $G$ be a 2-transitive permutation group of degree $n \geqslant 9$ on $\Omega$, and suppose that for $\alpha \in \Omega, G_{\alpha}$ has a system $\mathscr{B}$ of blocks of imprimitivity in $\Omega \backslash\{\alpha\}$ with blocks of size 2. Assume further that if $B=\{\beta, \gamma\} \in \Re_{3}$ then $G_{\alpha \beta \gamma}$ acts primitively on $\mathscr{B} \backslash\{B\}$. Then $n=9$ and $G$ is $A S L(2,3)$ or $A G L(2,3)$.

Proof. If $n=9$ the conclusion is easily seen to be true (see Sims (1970)), so assume that $n>9$. Let $B_{1}=\{\delta, \varepsilon\} \in \mathscr{B} \backslash\{B\}$ and write $L=G_{\alpha \beta \gamma}$. Now if $L_{\{\delta \varepsilon\}}$ has an orbit on $\mathscr{B} \backslash\left\{B, B_{1}\right\}$ of size 2 or 1 then since it is primitive, $L^{i B \backslash\{B\}}$ has order $p$ or $2 p$ where $p=\frac{1}{2}(n-3)$ is prime. But then $G$ contains an element which is a product of 1 or $2 p$-cycles and fixes at least 3 points, contradicting Theorem 13.10 of Wielandt (1964). Hence every orbit of $L_{\{\delta \varepsilon\}}$ on $\mathscr{B} \backslash\left\{B, B_{1}\right\}$ has size 3 or more, so $G_{\alpha \beta \gamma \delta \varepsilon}$ fixes no points in $\Omega \backslash\{\alpha, \beta, \gamma, \delta, \varepsilon\}$. This means that the unique third fixed point of $G_{\beta \delta}$ lies in $\{\alpha, \gamma, \varepsilon\}$, which is impossible as $G_{\beta \delta \alpha}, G_{\beta \delta \gamma}, G_{\beta \delta \varepsilon}$ fix $\gamma, \alpha, \alpha$ respectively.

Now let $G$ be primitive of degree $n$ on $\Omega$ and suppose that $G$ has a subgroup $H$ which has 2 nontrivial orbits $\Sigma_{1}, \Sigma_{2}$ of size at least 3 and acts primitively on both of them. If $n=9$ it is easy to see that (ii) of Theorem 2 holds, so we assume that $n>9$. To prove Theorem 2 it is enough to show that $G$ is 2 -primitive if $k \geqslant 2$ (where $k=\mid$ fix $H \mid$ ), for then the obvious induction argument will establish the result. Suppose then that $k \geqslant 2$ but $G$ is not 2-primitive. Let $\Sigma=\Sigma_{1} \cup \Sigma_{2}$ and define

$$
S=\left\{x \in G \mid \Sigma_{i} x \cap \Sigma \neq \varnothing \text { and } \Sigma_{i} x \nsubseteq \Sigma \text { for some } i \in\{1,2\}\right\}
$$

so that $\delta \neq \varnothing$ by Theorem 8.1 of Wielandt (1964). Pick $g \in \delta$ with $|\Sigma g \cup \Sigma|$ as small as possible. We may assume that $\Sigma_{1} g \cap \Sigma \neq \varnothing$ and $\Sigma_{1} g \not \Sigma \Sigma$.

STEP 2.1. We have $\Sigma_{2} g \cap \Sigma \neq \varnothing$. For suppose that $\Sigma_{2} g \cap \Sigma=\varnothing$. Now $\left|\Sigma_{1} g \cap \Sigma_{j}\right|>0$ for some $j \in\{1,2\}$. If $\left|\Sigma_{1} g \cap \Sigma_{j}\right| \geqslant 2$ choose $\alpha, \beta \in \Sigma_{1} g \cap \Sigma_{j}$. Since $H^{g}$ is primitive on $\Sigma_{1} g$ there exists $x \in H^{g}$ such that

$$
\alpha x \in \Sigma, \quad \beta x \in \Sigma_{1} g \backslash \Sigma \text {. }
$$

Then $x \in S$. But $\Sigma_{2} g \not \Sigma x$, so $|\Sigma \cup \Sigma x|<|\Sigma \cup \Sigma g|$, contradicting the choice of $g$. And if $\left|\Sigma_{1} g \cap \Sigma_{j}\right|=1$, say $\Sigma_{1} g \cap \Sigma_{j}=\{\alpha\}$, then any $x \in H^{g}$ with $\alpha x \in \Sigma_{1} g \backslash \Sigma$ contradicts the choice of $g$. 
Let $H_{1}=\left\langle H, H^{g}\right\rangle$. By Step 2.1, $H_{1}$ has $d$ nontrivial orbits $\Delta_{1}, \ldots, \Delta_{d}$ where $d$ is 1 or 2 . Write $\Theta_{i}=\Delta_{i} \backslash \Sigma(i=1, \ldots, d)$.

STEP 2.2. If $d=2$ then $\left|\Theta_{i}\right| \leqslant 1$ for $i=1,2$. We prove this for $i=1$; the case $i=2$ is covered by the same argument. Suppose first that $\left|\Theta_{1}\right| \geqslant\left|\Sigma_{1}\right|$. Then by choice of $g, \Sigma_{1}$ is a block for $H_{1}^{\Delta_{1}}$. This forces $\Sigma_{1} \subseteq \Sigma g$, for if not then there exists $x \in H^{g}$ fixing $\Sigma_{1} \backslash \Sigma g$ and mapping an element of $\Sigma_{1}$ to an element of $\Theta_{1}$, contradicting the fact that $\Sigma_{1}$ is a block for $H_{1}^{\Delta_{1}}$. However, since $H^{g}$ is primitive on $\Sigma_{i} g$ we have $\left|\Sigma_{1} \cap \Sigma_{i} g\right| \leqslant 1$ for $i=1,2$. This forces $\left|\Sigma_{1}\right| \leqslant 2$ as $\Sigma_{1} \subseteq \Sigma g$. This contradiction shows that $\left|\Theta_{1}\right|<\left|\Sigma_{1}\right|$. Hence by choice of $g, \Theta_{1}$ is a block for $H_{1}^{\Delta_{1}}$ and consequently $\left|\Theta_{1}\right| \leqslant 1$.

STEP 2.3. If $d=1$ then $\left|\Theta_{1}\right| \leqslant 2$ and $\Theta_{1}$ is a block for $H_{1}^{\Delta_{1}}$. To see this, suppose that $\left|\Theta_{1}\right| \geqslant 3$. Then $\left|\Sigma_{i} g \backslash \Sigma\right| \geqslant 2$ for some $i$, so since $H^{g}$ is primitive on $\Sigma_{i} g$ there exists $h \in H^{g}$ such that $\Theta_{1} h \neq \Theta_{1}$ and $\Theta_{1} h \cap \Theta_{1} \neq \varnothing$. By choice of $g$ it must be the case that $h \notin \delta$, so we may assume that

$$
\Sigma_{1} h \subseteq \Sigma \text { and } \Sigma_{2} h \subseteq \Theta_{1} .
$$

This implies that $\Sigma_{2} \subseteq \Sigma g$, and so

$$
\left|\Theta_{1}\right|=|\Sigma g|-|\Sigma g \cap \Sigma|=|\Sigma|-\left|\Sigma_{2}\right|-\left|\Sigma g \cap \Sigma_{1}\right|<\left|\Sigma_{1}\right| .
$$

First we show that $H_{1}^{\Delta_{1}}$ is primitive. Let $\Gamma$ be a block for $H_{1}^{\Delta_{1}}$ with $\Gamma \cap \Sigma_{2} \neq \varnothing$ and $|\Gamma|>1$. Now $\Gamma \cap \Sigma_{2}$ is a block for $H^{\Sigma_{2}}$, so either $\Sigma_{2} \subseteq \Gamma$ or $\left|\Gamma \cap \Sigma_{2}\right|=1$. If $\left|\Gamma \cap \Sigma_{1}\right|=1$ then $\Gamma \cap \Theta_{1}=\varnothing,\left|\Gamma \cap \Sigma_{1}\right|=1$ and $|\Gamma|=2$. This forces $\Gamma h(h$ as above) to contain a point of $\Sigma$ and a point of $\Theta_{1}$, which is impossible as $\Gamma h$ is a block for $H_{1}^{\Delta_{1}}$. Hence $\Sigma_{2} \subseteq \Gamma$, from which it follows without difficulty that $\Gamma=\Delta_{1}$, using the fact that $\Sigma_{j} g$ intersects both $\Sigma_{1}$ and $\Sigma_{2}$ nontrivially for some $j$ (since $d=1$ ).

Hence $H_{1}^{\Delta_{1}}$ is primitive. It follows from Theorem 1 that $H_{1}^{\Delta_{1}}$ is also 2-transitive (this can also be deduced here using elementary arguments).

Finally, choose $\alpha \in \Theta_{1}$. Since $H_{1}^{\Delta_{1}}$ is 2-transitive there exists $k \in H_{1 \alpha}$ such that $\Sigma_{1} k \cap \Theta_{1} \neq \varnothing$. Then certainly $\Sigma_{1} k \nsubseteq \Sigma$. Since $\left|\Theta_{1}\right|<\left|\Sigma_{1}\right|$ we have $\Sigma_{1} k \cap \Sigma \neq$ $\varnothing$. Hence $k \in \delta$. However, as $k \in H_{1 \alpha}$ we have

$$
\Sigma k \cup \Sigma \subseteq \Delta_{1} \backslash\{\alpha\}
$$

so that $k$ contradicts the choice of $g$.

This contradiction shows that $\left|\Theta_{1}\right| \leqslant 2$. Clearly by choice of $g, \Theta_{1}$ is a block for $H_{1}^{\Delta_{1}}$, so Step 2.3 is complete.

Note that in both cases $d=1$ and $d=2$ we have $|\Sigma g \backslash \Sigma| \leqslant 2$, so $g$ is such that $|\Sigma g \cup \Sigma|$ is minimal subject to the condition $\Sigma g \neq \Sigma$. In particular $\Sigma g \cup \Sigma \subset \Omega$ since $k \geqslant 2$, and so $\mid$ fix $H_{1} \mid \geqslant 1$. 
Suppose now that $d=1$, so that $\left|\Theta_{1}\right| \leqslant 2$ and $\Theta_{1}$ is a block for $H_{1}^{\Delta_{1}}$. Let $\Gamma$ be a proper block for $H_{1}^{\Delta_{1}}$ with $\Gamma \cap \Theta_{1} \neq \varnothing$. If $\Sigma_{1} \subseteq \Gamma$ or $\Sigma_{2} \subseteq \Gamma$ then $\Gamma=\Delta_{1}$ since $\Sigma_{i} g$ intersects both $\Sigma_{1}$ and $\Sigma_{2}$ for some $i$. Hence $\Gamma \subseteq \Theta_{1}$. Thus the only proper block systems for $H_{1}^{\Delta_{1}}$ are the trivial one and the one containing $\Theta_{1}$. If $\mid$ fix $H_{1} \mid \geqslant 2$ then Theorem I of Jordan (1871) (stated in the Introduction) shows that $G$ is 3-transitive, which is not so. Hence $\mid$ fix $H_{1} \mid=1$. But now if $\left|\Theta_{1}\right|=1$ then $H_{1}^{\Delta_{1}}$ is primitive, so $G$ is 2-primitive, which is not the case; and if $\left|\Theta_{1}\right|=2$ then Lemma 2.0 gives a contradiction.

Consequently $d=2$ and the orbits of $H_{1}$ are $\Sigma_{i}^{1}=\Sigma_{i} \cup \Theta_{i}$ where $\left|\Theta_{i}\right| \leqslant 1$ $(i=1,2)$. Write $\Sigma^{\prime}=\Sigma g \cup \Sigma$. Now define $g_{1}, g_{2}, \ldots$ inductively as follows: for $i=1,2, \ldots, g_{i} \in G$ is chosen such that $\left|\Sigma^{i} g_{i} \cup \Sigma^{i}\right|$ is minimal subject to the condition $\Sigma^{i} g_{i} \neq \Sigma^{i}$; and $\Sigma^{i+1}=\Sigma^{i} g_{i} \cup \Sigma^{i}$. By the above considerations the group $H_{i+1}=\left\langle H_{i}, H_{i}^{g_{i}}\right\rangle$ has 2 nontrivial orbits $\Sigma_{1}^{i+1}, \Sigma_{2}^{i+1}$. Clearly there is a positive integer $s$ such that $\left|\Omega \backslash \Sigma^{s}\right|=1$. Then fix $H_{s}=\{\alpha\}$, say, and $H_{s}$ has the 2 nontrivial orbits

$$
\Sigma_{i}^{s}=\Sigma_{i} \cup \Theta_{i} \cup \Theta_{i}^{\prime} \cup \cdots \cup \Theta_{i}^{s-1} \quad(i=1,2)
$$

where all $\left|\Theta_{i}^{j}\right| \leqslant 1$. If $u_{i}=\left|\Theta_{i} \cup \Theta_{i}^{1} \cup \cdots \cup \Theta_{i}^{s-1}\right|$ then $H_{s}$ is $\left(u_{i}+1\right)$-transitive on $\Sigma_{i}^{s}$. Since $k \geqslant 2$ we have $u_{1}+u_{2} \geqslant 1$.

We can now complete the proof of Theorem 2. If $G$ is not 2-transitive then $\Sigma_{1}^{s}$, $\Sigma_{2}^{s}$ are the nontrivial orbits of $G_{\alpha}$, and $G_{\alpha}$ cannot be 2-transitive on both of them by Result $\mathrm{A}$ (ii). Hence we may assume that $\Sigma_{1}^{s}=\Sigma_{1}$. But then $\Sigma_{1} \circ \Sigma_{2}^{s}$ is a suborbit of $G$ of size greater than $\left|\Sigma_{1}\right|$ and $\left|\Sigma_{2}^{s}\right|$ by Result $A(i)$, which is a contradiction.

Hence $G$ is 2-transitive. Finally, since $G$ is not 2-primitive, one of the following must hold:

(a) $\mathscr{B}=\left\{\Sigma_{1}^{s}, \Sigma_{2}^{s}\right\}$ is a block system for $G_{\alpha}$ on $\Omega \backslash\{\alpha\}$,

(b) $G_{\alpha}$ has a block system on $\Omega \backslash\{\alpha\}$ with blocks of size 2, each containing 1 point from $\Sigma_{1}^{s}$ and 1 from $\Sigma_{2}^{s}$.

In case (b) Lemma 2.0 gives a contradiction. In case (a) the kernel $K$ of the action of $G_{\alpha}$ on $\mathscr{B}$ contains $H_{s}$, so $K$ acts 2-transitively on both of its orbits $\Sigma_{i}^{s}(i=1,2)$. Hence by Theorem D of O'Nan (1975), $G$ is a normal extension of PSL $(m, q)$ in its natural 2-transitive representation on $P G(m-1, q)$ for some $m, q$. But the stabiliser of a point in such an extension of $\operatorname{PSL}(m, q)$ cannot have a block system with 2 blocks.

This contradiction completes the proof of Theorem 2 .

REMark. An obvious modification of the proof (in Step 2.2) shows that the restrictions $\left|\Sigma_{i}\right| \geqslant 3$ in Theorem 2 are unnecessary providing we exclude the groups $\operatorname{PSL}(2,7)$ of degree 7 and $A G L(3,2)$ of degree 8. 


\section{Some examples}

In this section we present some examples of primitive groups having subgroups with several fixed points and few nontrivial orbits.

EXAMPLE 3.1. Let $G$ be a group with $A S L(d, q) \leqslant G \leqslant A G L(d, q)$ acting on a $d$-dimensional vector space $V$ over $G F(q)(d \geqslant 2, q$ a prime power). Then the pointwise stabiliser $G_{(W)}$ of a $(d-1)$-dimensional subspace $W$ of $V$ acts semiregularly with degree $q^{d}-q^{d-1}$ on $V \backslash W$ and has $|A G L(d, q): G|$ nontrivial orbits. This number of orbits can be any positive integer dividing $q-1$; in particular it can be 2 (if $q$ is odd) or 3 (if $q \equiv 1(\bmod 3)$ ). Notice that the actions of $G_{(w)}$ on its orbits are primitive if and only if $d=2, q$ is prime and $G$ is $A S L(d, q)$; if $d=2$, $q=3$ then $G_{(W)}$ has 2 orbits of size 3 and 3 fixed points (see conclusion (ii) of Theorem 2).

EXAMPLE 3.2. Let $G$ be $P G L(d, q)(d \geqslant 3)$ acting on $P G(d-1, q)$, the set of 1-dimensional subspaces of $V$ and let $\left\{v_{1}, \ldots, v_{d}\right\}$ be a basis for $V$. For $r \leqslant s$ put $\Delta_{r s}=\left\{\langle v\rangle \mid\langle v\rangle \subseteq\left\langle v_{r}, \ldots, v_{s}\right\rangle\right\}$. Then $G_{\alpha \beta}$ has two nontrivial orbits for any $\alpha, \beta \in P G(d-1, q)$. For $s \leqslant d-1$ let $H_{s}$ be the subgroup $\left\{g \in G_{\left(\Delta_{1},\right)} \mid \Delta_{s+1 . d} g\right.$ $\left.\subseteq \Delta_{2 d}\right\}$. Then $H_{s}$ has two nontrivial orbits and fix $H_{s}=\Delta_{1 s}$.

Since ' $M_{21}$ ' is $\operatorname{PSL}(3,4)$ acting on $P G(2,4)$, considerations similar to the above give examples of subgroups of $M_{22}, M_{23}, M_{24}$ (in their representations of degrees $22,23,24$ respectively) having two nontrivial orbits of sizes either (a) 3 and 16 or (b) 8 and 8 .

EXAMPLE 3.3. As promised in the Introduction here is an example of a simply primitive group $G$ such that $G_{\alpha \beta}$ has 3 nontrivial orbits and acts primitively on each of them. Let $G$ be the Higman-Sims simple group $H S$ acting with degree 100 , as described in Higman and Sims (1968). Then $G$ has rank 3 and $G_{\alpha} \cong M_{22}$ has nontrivial orbits of sizes 22,77 . If $\beta$ is a point in the orbit of size 22 then $G_{\alpha \beta} \cong P S L(3,4)$ and $G_{\alpha \beta}$ has 3 nontrivial orbits of sizes 21,21 and 56 , acting 2 -transitively on the orbits of size 21 and primitively on that of size 56 .

In fact this is the only known example of a simply primitive group $G$ in which $G_{\alpha \beta}$ has 3 primitive orbits; for it is easy to see that such a group must have rank 3 and act 2-primitively on one of its suborbits. The known examples of such rank 3 groups are listed in Atkinson (1977) and $H S$ is the only one satisfying our requirements.

\section{Proof of Theorem 3}

As for the proof of Theorem 2 we require a preliminary lemma. 
LEMMA 4.0. Let $G$ be a 2-transitive group of degree $n \geqslant 13$ on $\Omega$ and suppose that $G_{\alpha}$ has a system $\cap$ of blocks of imprimitivity with blocks of size 3 satisfying the following conditions:

(i) if $B=\{\beta, \gamma, \delta\} \in$ in the $G_{\alpha \beta \gamma \delta}$ has a subgroup $H$ with 3 nontrivial orbits $\Sigma_{1}$, $\Sigma_{2}, \Sigma_{3}$ on $\Omega \backslash\{\alpha, \beta, \gamma, \delta\}$, each $H^{\Sigma_{i}}$ being primitive,

(ii) for any $B^{\prime} \in \mathscr{B} \backslash\{B\},\left|B^{\prime} \cap \Sigma_{i}\right|=1(i=1,2,3)$.

Then $n=13$ and $G$ is $\operatorname{PSL}(3,3)$.

Proof. If $n=13$ the conclusion is easily seen to be true (see Sims (1970)) so assume that $n \geqslant 14$. Let $B^{\prime}=\left\{\beta^{\prime}, \gamma^{\prime}, \delta^{\prime}\right\} \in \mathscr{B} \backslash\{B\}$ with $\beta^{\prime} \in \Sigma_{1}, \gamma^{\prime} \in \Sigma_{2}$, $\delta^{\prime} \in \Sigma_{3}$. Since $H \leqslant G_{\alpha}$ and $B^{\prime}$ is a block for $G_{\alpha}$ we have $H_{\beta^{\prime}}=H_{\gamma^{\prime}}=H_{\delta^{\prime}}$. By the argument in the proof of Lemma 2.0 every nontrivial orbit of $G_{\alpha \beta \gamma \delta \beta^{\prime} \gamma^{\prime} \delta^{\prime}}$ has size 3 or more. Now the $G$-translates of $\{\alpha\} \cup B$ form the blocks of a Steiner system $\delta(2,4, n)$ (see Case 1 on page 274 of Atkinson (1973)); however by the previous sentence the unique block containing $\left\{\beta, \beta^{\prime}\right\}$ must be contained in $\left\{\alpha, \beta, \gamma, \delta, \beta^{\prime}, \gamma^{\prime}, \delta^{\prime}\right\}$, which is impossible.

Now let $G$ be a primitive group of degree $n$ on $\Omega$ and suppose that $G$ has a subgroup $H$ which has 3 nontrivial orbits $\Sigma_{1}, \Sigma_{2}, \Sigma_{3}$ of size at least 4 and acts primitively on each of them. To prove Theorem 3 it is sufficient to show that $G$ is 2 -primitive if $k \geqslant 3$. Suppose then that $k \geqslant 3$ and $G$ is not 2-primitive. We mimic the proof of Theorem 2 . Thus let $\Sigma=\Sigma_{1} \cup \Sigma_{2} \cup \Sigma_{3}$ and define

$$
\S=\left\{x \in G \mid \Sigma_{i} x \cap \Sigma \neq \varnothing, \Sigma_{i} x \nsubseteq \Sigma \text { for some } i \in\{1,2,3\}\right\} .
$$

Choose $g \in \S$ with $|\Sigma g \cup \Sigma|$ minimal. Let $H_{1}=\left\langle H, H^{g}\right\rangle$ have nontrivial orbits $\Delta_{1}, \ldots, \Delta_{d}$ and write $\Theta_{i}=\Delta_{i} \backslash \Sigma(i=1, \ldots, d)$. Copying the proofs of Steps 2.1, 2.2 and 2.3 we have

STEP 4.1. We have $\Sigma_{i} g \cap \Sigma \neq \varnothing$ for $i=1,2,3$ (so $d \leqslant 3$ ). If $d=3$ then $\left|\Theta_{i}\right| \leqslant 1(i=1,2,3)$ and if $d=2$ then $\left|\Theta_{1}\right| \leqslant 2,\left|\Theta_{2}\right| \leqslant 1$ (where we take $\Delta_{1}$ to contain two of the $\Sigma_{i}$ ).

However the next step does not yield so easily.

STEP 4.2. If $d=1$ then $\left|\Theta_{1}\right| \leqslant 3$. Suppose that $\left|\Theta_{1}\right| \geqslant 4$. As in the proof of Step 2.3 there exists $h \in H^{g}$ such that $\Sigma_{m} h \subseteq \Theta_{1}$ and $\Sigma_{m} \subseteq \Sigma g$ for some $m \in\{1,2,3\}$. We may take $m=1$. Thus

$$
\Sigma_{1} h \subseteq \Theta_{1}, \quad \Sigma_{1} \subseteq \Sigma g
$$

Again it is easy to see that $H_{1}^{\Delta_{1}}$ is primitive, so by Jordan's Theorem $\Delta_{1}=\Omega$. 
We first show that $H_{1}$ is 2-transitive. Let $\alpha \in \Theta_{1}$ and let $\Gamma_{1}, \ldots, \Gamma_{s}$ be the orbits of $H_{1 \alpha}$ on $\Omega \backslash\{\alpha\}$. Suppose that $H_{1}$ is not 2-transitive, so that $s \geqslant 2$. For $i=1, \ldots, s$ let $B_{i}=\left\{j \mid \Sigma_{j} \subseteq \Gamma_{i}\right\}$. We obtain a contradiction in the following three stages.

(A) $\left|B_{i}\right| \geqslant 2$ for some $i$. For suppose that $\left|B_{i}\right| \leqslant 1$ for all $i$. Then by choice of $g$, if $j \in B_{i}$ then $\Sigma_{j}$ is a block for $H_{1 \alpha}^{\Gamma_{i}}$. Since $\left|\Theta_{1}\right|<|\Sigma|$ it follows that $\Gamma_{i}=\Sigma_{j}$ for some $i, j$. Choose $\Gamma_{k}$ of maximal size in

$$
\left\{\Gamma_{i} \mid \Gamma_{i}=\Sigma_{j} \text { for some } i, j\right\}
$$

and choose $\Gamma_{l}$ of maximal size in

$$
\left\{\Gamma_{i} \mid \Gamma_{i} \cap \Theta_{1} \neq \varnothing\right\} .
$$

Then by Result A, $\Gamma_{l}^{*} \circ \Gamma_{k}$ is a suborbit of $H_{1}$ of size greater than $\left|\Gamma_{l}\right|$ and $\left|\Gamma_{k}\right|$, which is a contradiction.

(B) If $j, k \in B_{i}$ for some $i$ then $\left|\Sigma_{j}\right|=\left|\Sigma_{k}\right|$. To see this, first suppose that $\left|B_{i}\right|=2$ and $\Gamma_{i} \cap \Theta_{1} \neq \varnothing$. Now if $\Sigma_{j} x \cap \Theta_{1} \neq \varnothing$ for some $x \in H_{1 \alpha}$ then $\Sigma_{j} x \subseteq \Theta_{1}$ by choice of $g$. Hence $H_{1 \alpha}^{\Gamma_{4}}$ is imprimitive and it is easy to see that $\Sigma_{j}$, $\Sigma_{k}$ are conjugate blocks for $H_{1 \alpha}^{\Gamma_{i}}$, so that $\left|\Sigma_{j}\right|=\left|\Sigma_{k}\right|$.

Next, if $\left|B_{i}\right|=3$ and $\Gamma_{i} \cap \Theta_{1} \neq \varnothing$ then either $\Sigma_{j}, \Sigma_{k}$ are conjugate blocks or $\Re=\left\{\Sigma_{i_{1}} \cup \Sigma_{i_{2}}, \Sigma_{i_{3}}, \Gamma_{i} \cap \Theta_{1}\right\}$ is a block system for $H_{1 \alpha}^{\Gamma_{i}}$ (where $\left\{i_{1}, i_{2}, i_{3}\right\}=$ $\{1,2,3\})$. In the latter case the kernel $K$ of the action of $H_{1 \alpha}$ on $\mathscr{B}$ contains $H$, so is primitive on $\Sigma_{i_{3}}$ and hence on $\Sigma_{i_{1}} \cup \Sigma_{i_{2}}$. Thus we may pick $y \in K$ such that

$$
\Sigma_{i_{1}} y \neq \Sigma_{i_{1}} \text { and } \Sigma_{i_{1}} y \cap \Sigma_{i_{1}} \neq \varnothing .
$$

Then $\left\langle H, H^{y}\right\rangle$ has only 2 nontrivial orbits $\Sigma_{i_{1}} \cup \Sigma_{i_{2}}$ and $\Sigma_{i_{3}}$, so $H_{1}$ is 2-transitive by Theorem 1, contradicting our assumption. If $\left|B_{i}\right|=3$ and $\Gamma_{i} \cap \Theta_{1}=\varnothing$ we obtain a similar contradiction unless $\Sigma_{j}, \Sigma_{k}$ are conjugate blocks for $H_{1 \alpha}^{\Gamma_{\alpha}}$.

Finally, if $\left|B_{i}\right|=2, \Gamma_{i} \cap \Theta_{1}=\varnothing$ and $\left|\Sigma_{j}\right| \neq\left|\Sigma_{k}\right|$ then $H_{1 \alpha}^{\Gamma_{i}}$ is primitive. The third orbit $\Sigma_{l}$ is contained in a block system $\mathscr{B}$ for $H_{1 \alpha}^{\Gamma_{f}}$ for some $f$, and the kernel $K$ of the action of $H_{1 \alpha}$ on $\mathscr{B}$ contains an element $y$ such that $\Sigma_{j} y \neq \Sigma_{j}$. Then $\left\langle H, H^{y}\right\rangle$ has only 2 nontrivial orbits and yields a contradiction by Theorem 1 again.

(C) For some $i, H^{\Sigma_{i}}$ is regular of prime degree. Since $\left|\Theta_{1}\right| \geqslant 4$ there exists $i \in\{1,23\}$ such that $\left|\Sigma_{i} g \backslash \Sigma\right| \geqslant 2$. Suppose that $H^{\Sigma_{i}}$ is not regular of prime degree and choose $\alpha \in \Sigma_{i} g \backslash \Sigma$. We first show that $\left|\Sigma_{1}\right|=\left|\Sigma_{2}\right|=\left|\Sigma_{3}\right|$. If $\left|B_{j}\right|=3$ for some $j$ this follows from (B), so we suppose (using (A)) that

$$
\left|B_{1}\right|=1, \quad\left|B_{2}\right|=2 .
$$

Assume first that $1 \in B_{1}$, that is, $\Sigma_{1} \subseteq \Gamma_{1}$. Recall that $\Sigma_{1} \subseteq \Sigma g$ and $h \in H^{g}$ is such that $\Sigma_{1} h \subseteq \Theta_{1}$. We have $\left|\Sigma_{2}\right|=\left|\Sigma_{3}\right|$ by (B). 
If $\Sigma_{2} \subseteq \Sigma g$ then

$$
\left|\Theta_{1}\right|=|\Sigma g|-|\Sigma g \cap \Sigma|<\left|\Sigma_{3}\right|
$$

so $\Gamma_{2}=\Sigma_{2} \cup \Sigma_{3}$ (for otherwise there exists $x \in H_{1 \alpha}$ such that $\Sigma_{3} x \subseteq \Theta_{1}$, which is impossible). Hence for $\beta \in \Theta_{1} \backslash\{\alpha\}$ we have

$$
\left(\Sigma_{2} \cup \Sigma_{3}\right) H_{1 \beta} \neq \Sigma_{2} \cup \Sigma_{3}
$$

since otherwise $\Sigma_{2} \cup \Sigma_{3}$ is a fixed set of $\left\langle H_{1 \alpha}, H_{1 \beta}\right\rangle=H_{1}$. Now (B) applied to the orbits of $H_{1 \beta}$ gives $\left|\Sigma_{1}\right|=\left|\Sigma_{2}\right|=\left|\Sigma_{3}\right|$.

If $\Sigma_{2} \nsubseteq \Sigma g, \Sigma_{3} \nsubseteq \Sigma g$ then either $\left(\Sigma_{2} \cup \Sigma_{3}\right)\left(H^{g}\right)_{\alpha}=\Sigma_{2} \cup \Sigma_{3}$ or (B) applied to the orbits of $H_{1 \alpha}$ gives $\left|\Sigma_{1}\right|=\left|\Sigma_{2}\right|=\left|\Sigma_{3}\right|$; so suppose $\left(\Sigma_{2} \cup \Sigma_{3}\right)\left(H^{g}\right)_{\alpha}=\Sigma_{2} \cup$ $\Sigma_{3}$. Let $\beta \in \Sigma_{i} g \backslash \Sigma$ with $\beta \neq \alpha$. Since we have assumed that $\left(H^{g}\right)^{\Sigma_{i} g}$ is not regular we have

$$
\left(H^{g}\right)^{\Sigma_{i} g}=\left\langle\left(H^{g}\right)_{\alpha},\left(H^{g}\right)_{\beta}\right\rangle^{\Sigma_{i g}} .
$$

It is easy to see that $\Sigma_{i} g$ must intersect $\Sigma_{2} \cup \Sigma_{3}$ nontrivially, so

$$
\left(\Sigma_{2} \cup \Sigma_{3}\right)\left(H^{g}\right)_{\beta} \neq \Sigma_{2} \cup \Sigma_{3} \text {. }
$$

Now (B) applied to the orbits of $H_{1 \beta}$ gives $\left|\Sigma_{1}\right|=\left|\Sigma_{2}\right|=\left|\Sigma_{3}\right|$.

We have now shown that $\left|\Sigma_{1}\right|=\left|\Sigma_{2}\right|=\left|\Sigma_{3}\right|$ if $1 \in B_{1}$. Similar arguments yield the same conclusion if $2 \in B_{1}$ or $3 \in B_{1}$. Hence in all cases,

$$
\left|\Sigma_{1}\right|=\left|\Sigma_{2}\right|=\left|\Sigma_{3}\right| \text {. }
$$

Now we may choose $\alpha\left(\in \Sigma_{i} g \backslash \Sigma\right)$ such that $\Sigma H_{1 \alpha} \neq \Sigma$. We have

$$
\left|\Theta_{1}\right|=|\Sigma g|-|\Sigma g \cap \Sigma|<|\Sigma g|-\left|\Sigma_{1}\right|=2\left|\Sigma_{1}\right| \text {. }
$$

By the argument of Step 1.3 any nontrivial orbit of $H_{1 \alpha}$ has size at least $\left|\Sigma_{1}\right|$. Hence since $\left|\Theta_{1}\right|<2\left|\Sigma_{1}\right|$ and $\Sigma H_{1 \alpha} \neq \Sigma, H_{1 \alpha}$ must have precisely 2 nontrivial orbits $\Gamma_{1}, \Gamma_{2}$ with either

(1) $\Sigma_{i_{1}}=\Gamma_{1}, \Sigma_{i_{2}} \cup \Sigma_{i_{3}} \subset \Gamma_{2}$, or

(2) $\Sigma_{i_{1}} \subset \Gamma_{1}, \Sigma_{i_{2}} \cup \Sigma_{i_{3}}=\Gamma_{3}$

where $\left\{i_{1}, i_{2}, i_{3}\right\}=\{1,2,3\}$. In case (1) the suborbit $\Gamma_{2} \circ \Gamma_{1}$ gives the usual contradiction by Result A. In case (2), for any $\beta \in \Gamma_{1} \backslash \Sigma_{i}$, one of the following possibilities must hold:

(a) the orbits of $H_{1 \beta}$ are as in case (1) above,

(b) $H_{1 \beta}$ has an orbit $\Sigma_{i} \cup \Sigma_{j}$ for some $i, j$,

(c) $\Sigma H_{1 \beta}=\Sigma$.

Since $\left\langle H_{1 \alpha}, H_{1 \beta}\right\rangle=H_{1}$ for all $\beta \in \Gamma_{1} \backslash \Sigma_{i_{1}}$ and $\left|\Gamma_{1} \backslash \Sigma_{i_{1}}\right| \geqslant\left|\Sigma_{i_{1}}\right| \geqslant 4$, it is clear that (a) must hold for some $\beta$. This gives a contradiction as in case (1).

This finishes the proof of $(\mathrm{C})$.

Now we can complete the proof of the 2-transitivity of $H_{1}$. By (C), some $H^{\Sigma_{i}}$ is regular of prime degree $p$, say. Let $K$ be the kernel of the action of $H$ on $\Sigma_{i}$. If 
$K \neq 1$ then by Theorem 8.8 of Wielandt (1964), $K$ has at most 2 nontrivial orbits, so $H_{1}$ is 2-transitive by Theorem 1 . And if $K=1$ then $|H|=p$ and so $G$ contains an element which is a product of 1,2 or $3 p$-cycles, contradicting Theorem 13.10 of Wielandt (1964).

Hence $H_{1}$ is 2-transitive. We obtain a final contradiction, proving Step 4.2, by showing that $H_{1}$ is 2-primitive. It is easy to see that the only possible proper, nontrivial block systems $\mathscr{Q}$ for $H_{1 \alpha}\left(\alpha \in \Theta_{1}\right)$ are

$$
\begin{gathered}
\left\{\Sigma_{1}, \Sigma_{2}, \Sigma_{3}, \Theta_{1} \backslash\{\alpha\}\right\}, \quad\left\{\Sigma_{i_{1}} \cup \Sigma_{i_{2}}, \Sigma_{i_{3}}, \Theta_{1} \backslash\{\alpha\}\right\}, \\
\left\{\Sigma_{i_{1}} \cup \Sigma_{i_{2}} \cup \Theta_{1} \backslash\{\alpha\}, \Sigma_{i_{3}}\right\}
\end{gathered}
$$

where $\left\{i_{1}, i_{2}, i_{3}\right\}=\{1,2,3\}$. In each case let $K$ be the kernel of the action of $H_{1 \alpha}$ on $\%$. In the first two cases $K$ does not restrict faithfully to its orbits on $\Omega \backslash\{\alpha\}$, and in the third case $K$ is 2-transitive on each of its orbits (by Theorem 1). Now Theorem D and Proposition 4 of O'Nan (1975) show that none of these cases is possible.

This completes Step 4.2.

Note that in each of the cases $d=1, d=2, d=3$ we have $|\Sigma g \backslash \Sigma| \leqslant 3$, so $g$ is such that $|\Sigma g \cup \Sigma|$ is minimal subject to the condition $\Sigma g \neq \Sigma$. Hence $\Sigma g \cup \Sigma$ $\subset \Omega$ since $k \geqslant 3$, and so $\mid$ fix $H_{1} \mid \geqslant 1$.

The argument presented after Step 2.3 (using Lemma 4.0 instead of Lemma 2.0) shows that $d$ cannot be 1 . Consequently either $d=3$ and $H_{1}$ has orbits

$$
\Sigma_{i}^{1}=\Sigma_{i} \cup \Theta_{i} \quad\left(\left|\Theta_{i}\right| \leqslant 1, i=1,2,3\right)
$$

or $d=2$ and we may take $H_{1}$ to have orbits

$$
\Sigma_{1}^{1}=\Sigma_{1} \cup \Sigma_{2} \cup \Theta_{1} \quad \text { and } \quad \Sigma_{2}^{l}=\Sigma_{3} \cup \Theta_{2} \quad\left(\left|\Theta_{1}\right| \leqslant 2,\left|\Theta_{2}\right| \leqslant 1\right) .
$$

Write $\Sigma^{1}=\Sigma g \cup \Sigma$. Now for $i=1,2, \ldots$ choose $g_{i} \in G$ such that $\left|\Sigma^{i} g_{i} \cup \Sigma^{i}\right|$ is minimal subject to the condition $\Sigma^{i} g_{i} \neq \Sigma^{i}$. Write $\Sigma^{i+1}=\Sigma^{i} g_{i} \cup \Sigma^{i}$ and $H_{i+1}=$ $\left\langle H_{i}, H_{i}^{g_{1}}\right\rangle$. For some $r$ we have $\Sigma^{r}=\Omega \backslash\{\alpha\}$ for some $\alpha \in \Omega$. Then by Steps 4.1, 4.2 and the above reasoning (note however that we must use an obvious modification of Lemma 4.0 if some $H_{i}$ has only 2 nontrivial orbits), the nontrivial orbits of $H_{r}$ are of one of the following types:

(i) 3 orbits $\Sigma_{i}^{r}=\Sigma_{i} \cup \Theta_{i} \cup \Theta_{i}^{l} \cup \cdots \cup \Theta_{i}^{r-1}\left(i=1,2,3\right.$, all $\left.\left|\Theta_{i}^{j}\right| \leqslant 1\right)$,

(ii) 2 orbits $\Sigma_{1}^{r}=\Sigma_{1} \cup \Sigma_{2} \cup \Theta_{1} \cup \Theta_{1}^{1} \cup \cdots \cup \Theta_{1}^{r-1}$ and $\Sigma_{2}^{r}=\Sigma_{3} \cup \Theta_{2} \cup \Theta_{2}^{\prime} \cup \cdots \cup \Theta_{2}^{r-1}, \quad\left(\right.$ all $\left.\left|\Theta_{1}^{\prime}\right| \leqslant 2,\left|\Theta_{2}^{j}\right| \leqslant 1,\left|\Theta_{1}\right| \leqslant 2,\left|\Theta_{2}\right| \leqslant 1\right)$.

STEP 4.3. $G$ is 2-transitive. Suppose false; then the rank of $G$ is 3 or 4 . If it is 4 then the nontrivial orbits of $G_{\alpha}$ are $\Sigma_{i}^{r}(i=1,2,3)$ and by Result $\mathrm{A}(\mathrm{ii}), \Sigma_{i}^{r}=\Sigma_{i}$ for some $i$. Hence if $\Sigma_{j}$ is of maximal size in $\left\{\Sigma_{i} \mid \Sigma_{i}^{r}=\Sigma_{i}\right\}$ and $\Sigma_{k}^{r}$ is of maximal 
size in $\left\{\Sigma_{i}^{r} \mid \Sigma_{i} \subset \Sigma_{i}^{r}\right\}$ then by Result A(i), $\Sigma_{j}^{*} \circ \Sigma_{k}^{r}$ is a suborbit of $G$ of size greater than $\left|\Sigma_{j}\right|$ and $\left|\Sigma_{k}^{r}\right|$, which is impossible. Consequently $G$ has rank 3 and $G_{\alpha}$ has as orbits either

$$
\begin{aligned}
& \Gamma_{1}=\Sigma_{1}^{r} \cup \Sigma_{2}^{r}, \Gamma_{2}=\Sigma_{3}^{r}\left(\Sigma_{i}^{r}\right. \text { as in (i) above), or } \\
& \Gamma_{1}=\Sigma_{1}^{r}, \Gamma_{2}=\Sigma_{2}^{r}\left(\Sigma_{i}^{r}\right. \text { as in (ii) above). }
\end{aligned}
$$

We suppose that the first case holds; it will easily be seen that the ensuing argument also applies to the second case.

Thus we are assuming that $\Gamma_{1}=\Sigma_{1}^{r} \cup \Sigma_{2}^{r}, \Gamma_{2}=\Sigma_{2}^{r}$. Write $\Theta_{i}^{0}=\Theta_{i}$ and let

$$
\Delta_{i}=\Sigma_{i}^{r} \backslash \Theta_{i}^{r-1} \text { and } d_{i}=\left|\Delta_{i}\right| \quad(i=1,2,3) .
$$

Since $H_{r}$ is 2-transitive on some $\Sigma_{i}^{r},|G|$ is even and so $\Gamma_{1}, \Gamma_{2}$ are self-paired suborbits of $G$. We deal separately with the cases $I .\left|\Theta_{1}^{r-1} \cup \Theta_{2}^{r-1}\right|>0$ and II. $\left|\Theta_{1}^{r-1} \cup \Theta_{2}^{r-1}\right|=0$.

I. The case $\left|\Theta_{1}^{r-1} \cup \Theta_{2}^{r-1}\right|>0$. Choose $\gamma \in \Theta_{1}^{r-1}$, say. If $\left|\Theta_{3}^{r-1}\right|=0$ then $G_{\alpha \gamma}$ is transitive on $\Gamma_{2}$, giving the usual contradiction by Result A. Hence $\Theta_{3}^{r-1}=\{\beta\}$, say. If $\beta \in \Gamma_{2}(\delta)$ for some $\delta \in \Delta_{3}$ then, since $G_{\alpha \beta}$ is transitive on $\Delta_{3}$ we have $\beta \in \Gamma_{2}(\delta)$ for all $\delta \in \Delta_{3}$ and $\Gamma_{2} \cup\{\alpha\}$ is a component in the $\Gamma_{2}$-graph, which is impossible by Lemma 3 of Neumann (1977). Hence the $\Gamma_{2}$-graph has no triangles. If $\gamma \in \Gamma_{2}(\delta)$ for all $\delta \in \Delta_{3}$ then since the $\Gamma_{1}$-graph is connected, we have $\gamma \in \Gamma_{1}(\beta)$; since $\left|\Gamma_{2}(\gamma)\right|=\left|\Delta_{3}\right|+1$ it follows that $\Theta_{2}^{r-1}=\{\varepsilon\}$, say, and $\gamma \in \Gamma_{2}(\varepsilon)$. But then $\gamma, \delta, \varepsilon$ form a triangle in the $\Gamma_{2}$-graph for any $\delta \in \Delta_{3}$, which is not so. Thus $\gamma \in \Gamma_{1}(\delta)$ for all $\delta \in \Delta_{3}$ and so $\gamma \in \Gamma_{2}(\beta)$. Now for some $i \in\{1,2\}$ we have $\beta \in \Gamma_{2}(\delta)$ for all $\delta \in \Delta_{i}$; we may take $i=1$. Hence, since there are no triangles in the $\Gamma_{2}$-graph, $\gamma \in \Gamma_{2}(\delta)$ for all $\delta \in \Delta_{2}$. Consequently $\left|\Theta_{2}^{r-1}\right|=0$; for if $\Theta_{2}^{r-1}=\{\varepsilon\}$, say, then $\gamma, \varepsilon$ have at least $d_{2}$ mutual adjacencies in the $\Gamma_{2}$-graph (the points of $\Delta_{2}$ ), whereas $\alpha, \gamma$ have only 1 mutual adjacency (the point $\beta$ ). Since $\gamma \in \Gamma_{1}(\varepsilon)$ and $\gamma \in \Gamma_{1}(\alpha)$ this is a contradiction. Thus, writing $v_{2}(\beta)$ for the valency of $\beta$ in the $\Gamma_{2}$-graph, we have

$$
\left|\Gamma_{2}\right|=d_{3}+1=v_{2}(\beta)=d_{1}+2=v_{2}(\gamma)=d_{2}+1 .
$$

Counting edges in the $\Gamma_{2}$-graph between $\Gamma_{2}$ and $\Gamma_{1}$, we obtain

$$
\left|\Gamma_{2}\right|\left(d_{1}+1\right)=\left|\Gamma_{1}\right| \text {. }
$$

Since $\left|\Gamma_{1}\right|=d_{1}+d_{2}+1$ this forces $d_{3}$ to be 1 or 0 , which is not so.

II. The case $\left|\Theta_{1}^{r-1} \cup \Theta_{2}^{r-1}\right|=0$. Certainly $\left|\Theta_{3}^{r-1}\right|=1$ here, say $\Theta_{3}^{r-1}=\{\beta\}$. Since $\mid$ fix $H \mid=k \geqslant 3$ we have

$$
\bigcup_{i=1}^{3} \Theta_{i}^{r-2} \neq \varnothing
$$

Suppose first that $\left|\Theta_{1}^{r-2} \cup \Theta_{2}^{r-2}\right|>0$, say $\Theta_{1}^{r-2}=\{\gamma\}$. If $\gamma \in \Gamma_{2}(\delta)$ for $\delta \in$ $\Delta_{3} \backslash \Theta_{3}^{r-2}$ then since $v_{2}(\gamma)$ is $d_{3}+1$ or $d_{3}+2$, we have $\Theta_{2}^{r-2}=\{\varepsilon\}$, say, and 
$\gamma \in \Gamma_{2}(\varepsilon)$. Then $\gamma, \delta, \varepsilon$ form a triangle in the $\Gamma_{2}$-graph for any $\delta \in \Delta_{3} \backslash \Theta_{3}^{r-2}$, which cannot be so. Hence $\gamma \in \Gamma_{1}(\delta)$ for all $\delta \in \Delta_{3} \backslash \Theta_{3}^{r-2}$. If $\gamma \in \Gamma_{2}(\beta)$ then $\beta \in \Gamma_{2}(\delta)$ for all $\delta \in \Delta_{1}$, so $\gamma \in \Gamma_{2}(\delta)$ for all $\delta \in \Delta_{2} \backslash \Theta_{2}^{r-2}$ and we have

$$
\left|\Gamma_{2}\right|=d_{3}+1=v_{2}(\beta)=d_{1}+1=v_{2}(\gamma)
$$

and $v_{2}(\gamma)$ is one of $d_{2}, d_{2}+1$ and $d_{2}+2$. However $\left|\Gamma_{2}\right| d_{1}$ is either $\left|\Gamma_{1}\right|$ or $2\left|\Gamma_{1}\right|$, which forces $d_{1} \leqslant 3$, a contradiction. Thus $\gamma \in \Gamma_{1}(\beta)$; this yields a similar contradiction.

Finally, suppose that $\left|\Theta_{1}^{r-2} \cup \Theta_{2}^{r-2}\right|=0$ and let $\Theta_{3}^{r-2}=\{\varepsilon\}$. We may assume that $\beta \in \Gamma_{2}(\delta)$ for all $\delta \in \Delta_{1}$ and $\beta \in \Gamma_{1}(\delta)$ for all $\delta \in \Delta_{2}$. Hence

$$
\Delta_{i} G_{\alpha \beta}=\Delta_{i} \quad \text { for } i=1,2 \text {. }
$$

Now some points of $\Delta_{2}$ are joined to points of $\Delta_{3}$ in the $\Gamma_{2}$-graph, so $\varepsilon \in \Gamma_{2}(\delta)$ for all $\delta \in \Delta_{2}$. From the action of $G_{\alpha \beta}$ we see that $\gamma \in \Gamma_{2}(\delta)$ for all $\gamma \in \Delta_{3}$, $\delta \in \Delta_{2}$. Pick $\delta_{1} \in \Delta_{1}, \delta_{2} \in \Delta_{2}$. Then $\alpha, \delta_{1}$ have 1 mutual adjacency in the $\Gamma_{2}$-graph, but $\alpha, \delta_{2}$ have $d_{3}$ mutual adjacencies, which is a contradiction.

This completes Step 4.3.

Now we can finish the proof of Theorem 3. Suppose that the orbits of $H_{r}$ are of type (i) described above (just before Step 4.3). Now $G_{\alpha}$ is imprimitive on $\Omega \backslash\{\alpha\}$ by assumption. Let $\Delta$ be a proper, nontrivial block for $G_{\alpha}$. If $|\Delta|<4$ then it is easy to see that $|\Delta|=3$ and Lemma 4.0 gives a contradiction. And if $|\Delta| \geqslant 4$ we can take the block system $B$ containing $\Delta$ to be one of

$$
\left\{\Sigma_{1}^{r}, \Sigma_{2}^{r}, \Sigma_{3}^{r}\right\} \text { and }\left\{\Sigma_{1}^{r} \cup \Sigma_{2}^{r}, \Sigma_{3}^{r}\right\} \text {. }
$$

Let $K$ be the kernel of the action of $G_{\alpha}$ on $\mathscr{B}$, so that $H_{r} \leqslant K$. Then (using Theorem 1 in the second case) $K$ is 2-transitive on each of its orbits and we have a contradiction by Theorem D of O'Nan (1975).

Similar arguments deal with the case where the orbits of $H_{r}$ are of type (ii). Thus Theorem 3 is proved.

Remark. Again (see the remark at the end of Section 2) we can relax the restrictions in Theorem 3 to $\left|\Sigma_{i}\right| \geqslant 3$, providing we exclude the group $\operatorname{PSL}(3,3)$ of degree 13.

\section{References}

V. D. Antopolski (1971), 'A certain condition for double transitivity of a primitive permutation group', Math. USSR-Sb. 14, 582-586.

M. D. Atkinson (1973), 'Two theorems on doubly transitive permutation groups', J. London Math. Soc. 6, 269-274. 
M. D. Atkinson (1977), 'On rank 3 groups having $\lambda=0$ ', Canad. J. Math. 29, 845-847.

D. G. Higman and C. C. Sims (1968), 'A simple group of order 44,352,000', Math. Z. 105, 110-13.

C. Jordan (1971), 'Théorèmes sur les groupes primitifs', J. Math. Pures Appl. (Liouville) 16, 383-408.

M. W. Liebeck (1977), Primitive permutation groups: a problem of Wielandt (M. Sc. Dissertation, Oxford).

B. Marggraff (1892), Über primitive Gruppen mit transitiven Untergruppen geringeren Grades (Dissertation, Giessen).

P. M. Neumann (1977), 'Finite permutation groups, edge-coloured graphs and matrices', Topics in group theory and computation, Proc. Summer School (Galway 1973), pp. 82-118, (London, Academic Press).

M. E. O'Nan (1975), 'Normal structure of the one-point stabiliser of a doubly transitive permutation group. II ', Trans. Amer. Math. Soc. 214, 43-74.

C. C. Sims (1970), 'Computational methods in the study of permutation groups', Computational problems in abstract algebra, Proc. Conf. (Oxford 1967), ed. J. Leech, pp. 169-183. (Pergamon Press, Oxford-New York).

H. Wielandt (1971), Subnormal subgroups and permutation groups (Lecture notes, Ohio State University).

H. Wielandt (1964), Finite permutation groups (Academic Press, London-New York).

Department of Pure Mathematics

University College

Cardiff CF1 $1 \mathrm{XL}$, Wales

U. $\mathrm{K}$. 